\title{
Angiogenesis: a phenomenon which aggravates chronic liver disease progression
}

\author{
Michał Kukla
}

Received: 21 February 2012/ Accepted: 21 June 2012/Published online: 26 July 2012

(C) Asian Pacific Association for the Study of the Liver 2012

\begin{abstract}
It has become increasingly clear that angiogenesis occurring during chronic wound healing and fibrogenesis provides a key contribution to disease progression and complications. The association of fibrogenesis and angiogenesis should be regarded as crucial in the modern evaluation of liver disease progression and in the search for therapeutic targets. Physiological hepatic angiogenesis occurs during liver regeneration, contributing to the formation of new functional sinusoids. Pathological angiogenesis in liver is characterized by intrahepatic vascular remodeling with capillarization of the sinusoids and development of intrahepatic shunts, which lead to increased hepatic resistance and decreased effective hepatocyte perfusion. The problem of angiogenesis in chronic hepatitis $\mathrm{C}$ and nonalcoholic fatty liver disease has not been fully resolved. This manuscript briefly describes pathogenesis of new blood vessel formation in chronic hepatitis and potential role of angiogenesis in disease progression.
\end{abstract}

Keywords Adipokine - Angiogenesis - Chronic hepatitis C · Fibrosis - Hepatic stellate cell · Inflammatory activity · Liver $\cdot$ Nonalcoholic fatty liver disease

\section{Introduction}

Angiogenesis, the formation of new vascular structures from preexisting vessels, occurs in several organs during multiple pathophysiological conditions [1]. Although

M. Kukla ( $\square)$

Department of Physiology in Zabrze, Medical University of Silesia in Katowice, Ul. Jordana 19, 41-800 Zabrze, Poland e-mail: kuklamich@poczta.onet.pl angiogenesis is an integral part of tumor progression, it has also been observed in different inflammatory, fibrotic, and ischemic diseases [2]. Chronic liver diseases (CLDs) do not represent an exception to this rule. The suggestion that angiogenesis may significantly contribute to fibrogenesis and disease progression relies first on the fact that vascular remodeling is a common finding in human liver with advanced fibrosis, irrespective of etiology [3-7].

In the current issue of Hepatology International, Ciupińska-Kajor et al.'s [7] very interesting study suggests that the amount of visceral adipose tissue may influence the development of liver fibrosis in nonalcoholic fatty liver disease (NAFLD), and that early angiogenesis might be the distinguishing feature. An intriguing finding of the current manuscript is the presence of angiogenesis and development of fibrosis in morbidly obese patients with simple steatosis, whereas in nonobese subjects both these morphological features are observed in the case of nonalcoholic steatohepatitis (NASH). Another interesting finding is that there was a positive relationship between the stage of fibrosis and angiogenic activity in morbidly obese patients. The study assessed liver expression of angiogenic markers-vascular endothelial growth factor (VEGF), VEGF receptor 2-kinase insert domain receptor (VEGF-R2 or Flk-1) and CD 34. Liver expression of these angiogenic markers was higher in obese compared to nonobese patients with simple steatosis or non-defined NASH. In the case of defined NASH, there was no difference in expression of angiogenic markers between morbidly obese and nonobese patients.

During the pathogenesis of CLDs, neovascularization and the establishment of fibrosis and abnormal angioarchitecture are related to their pathological progression. The association of fibrogenesis and angiogenesis should be regarded as crucial in the modern evaluation of liver disease progression and in the search for therapeutic targets. It 
is still unclear whether angiogenesis merely represents a homeostatic mechanism aimed at ensuring an adequate oxygen supply or one that exerts an additional pathogenic role leading to liver damage $[1,3]$. The accumulation of inflammatory cells and development of fibrosis may increase the resistance of liver tissue to blood flow and oxygen supply, resulting into hypoxia [3]. Hypoxia promotes angiogenesis as a consequence of signaling mediated by hypoxia-inducible factors (HIFs) that are transcription factors $[8,9]$. Hypoxia inhibits the hydroxylation of HIF$1 \alpha$, and therefore, decreases its degradation by proteasome. This results in HIF- $1 \alpha$ stabilization and accumulation in the cytosol. Subsequently, HIF- $1 \alpha$ joins with the $\beta$-subunit, which triggers translocation to the nucleus and modulates the expression of various genes that promote angiogenesis [9]. Liver tissue injury leads to an immunological response, in which immune cells must extravasate into the damaged tissue. Persistent inflammation facilitates vascular permeability and enhances the chemokine-mediated recruitment of macrophages, monocytes, platelets, and mast cells into areas of inflammation [10]. These circumstances provoke proliferation and migration of endothelial cells (ECs) with subsequent development of new blood vessels [8, 11, 12]. Angiogenesis in liver is characterized by intrahepatic vascular remodeling with capillarization of the sinusoids and development of intrahepatic shunts, which lead to increased hepatic resistance and decreased effective hepatocyte perfusion [1, 3]. It has become increasingly clear that angiogenesis occurring during chronic wound healing and fibrogenesis provides a key contribution to disease progression and complications [11, 13]. Two main pathways responsible for angiogenesis development in fibrogenic CLDs are shown in Fig. 1.

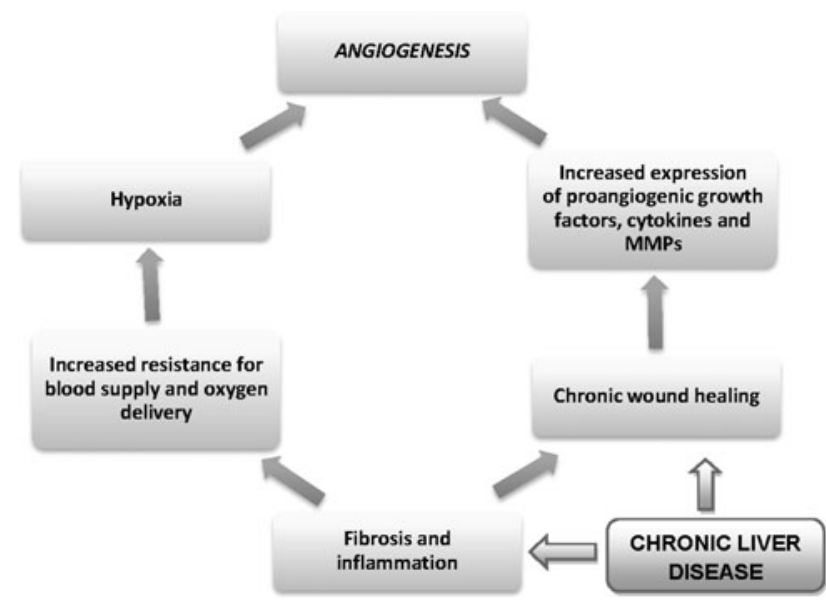

Fig. 1 Two main pathways responsible for angiogenesis development in fibrogenic chronic liver diseases

\section{Phases of angiogenesis and the molecules involved}

The formation of new blood vessels requires precise regulation. It encompasses several phases and involves a broad spectrum of proangiogenic agents. Consecutive phases of new blood vessel formation are shown in Fig. 2. The agents involved and their role in angiogenesis development are described in Fig. 3.

Sprouting and budding start with the establishment of conditions that allow ECs proliferation and migration. Plasma proteins must leak from the existing vessels. These proteins together with extracellular matrix (ECM) components serve as a provisional scaffold for EC migration. Nitric oxide (NO) whose angiogenic properties have been described induces vasodilatation [14], whereas VEGF increases vascular permeability [1]. Next, ECM must be degraded to allow EC migration and proliferation. ECM degradation results from the increased activity of various specialized proteinases such as matrix metalloproteinases (MMPs), urokinase plasminogen activator (uPA), and their corresponding inhibitors-tissue inhibitors of metalloproteinases (TIMPs) and plasminogen activator inhibitor-1 (PAI-1) [15]. ECM proteolysis leads to the exposure of cryptic epitopes and release of ECM-embedded factors that promote EC migration and proliferation. $\alpha \mathrm{v} \beta 3$ and $\alpha \mathrm{v} \beta 5$ integrins regulate the detachment/attachment of ECs to the ECM and maintain communication between the EC and its neighborhood [16]. Insufficient or inadequate proteolysis precludes EC migration [17], whereas excessive ECM degradation destabilizes the support structure on which ECs migrate, thereby inhibiting angiogenesis [18].

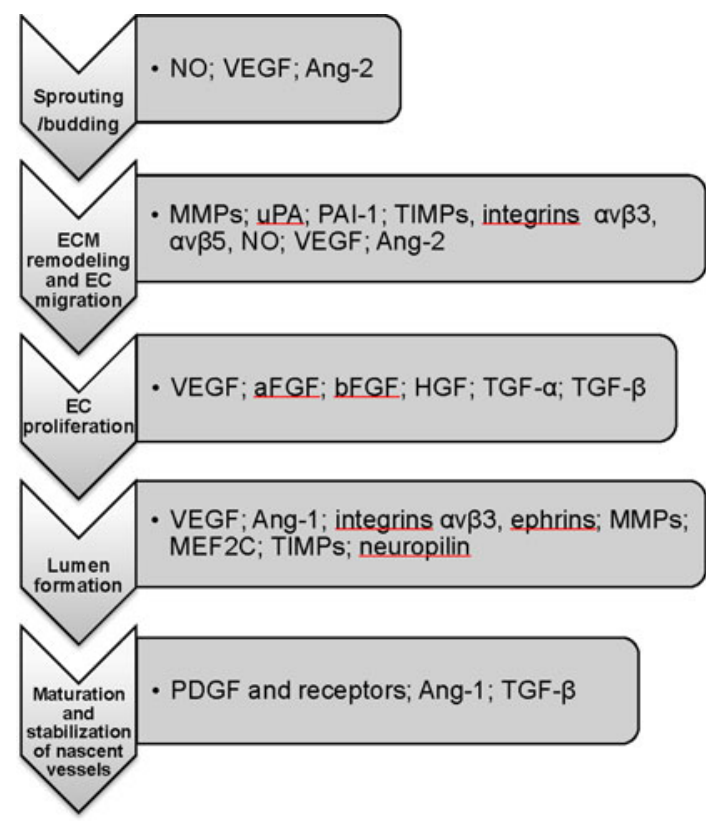

Fig. 2 Phases of angiogenesis and the agents involved 
Fig. 3 Proangiogenic molecules and their action

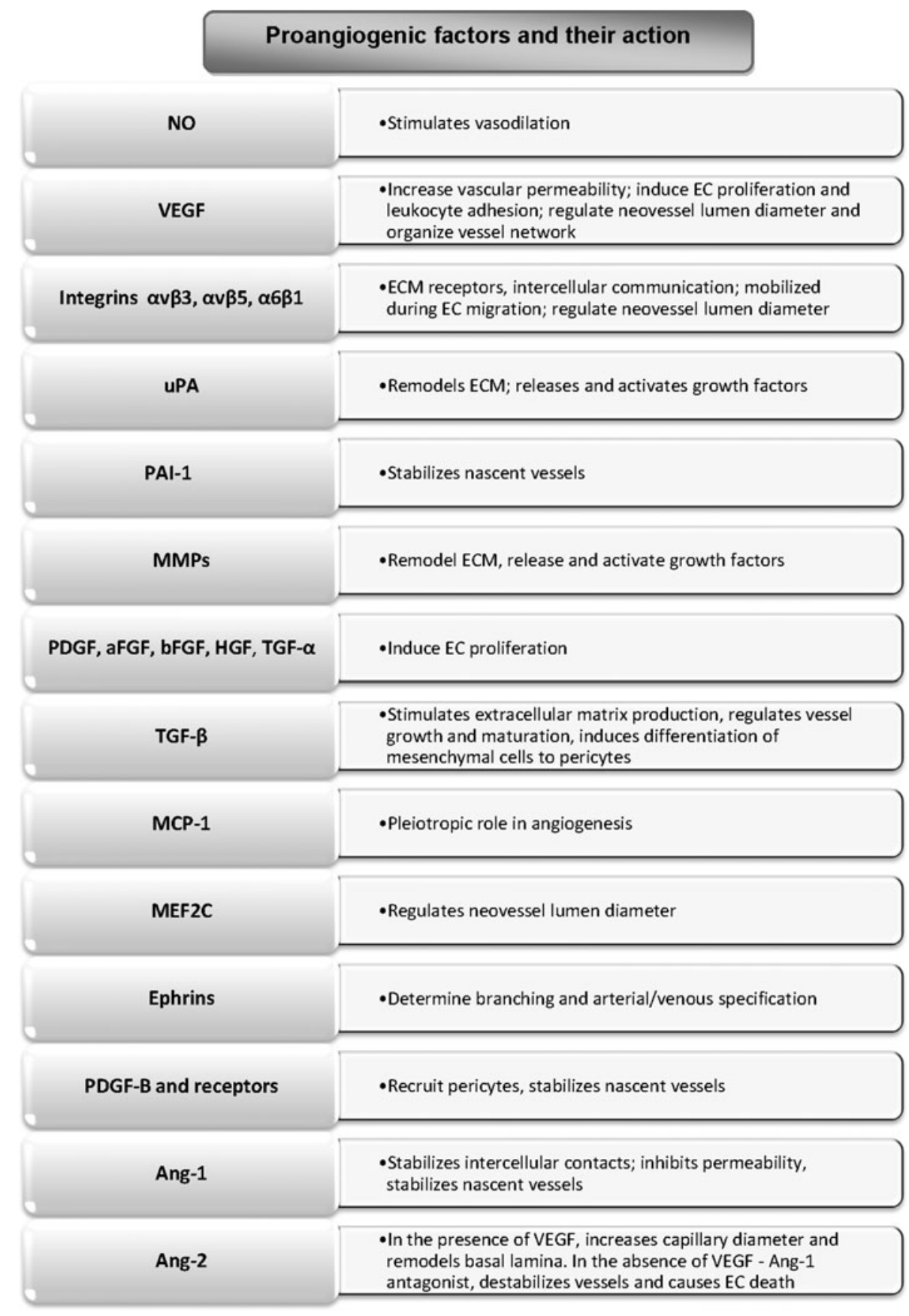

EC proliferation occurred in response to growth factors secreted by ECs or surrounding cells, including hepatic stellate cells (HSCs), leukocytes, hepatocytes, and Kupffer cells [19]. The well-characterized proangiogenic factor is VEGF, a multifunctional protein that binds to two tyrosine kinase receptors: FMS-like tyrosine kinase receptor (VEGF-R1 or FLT-1) and VEGF-R2 or Flk-1 [19]. Additionally, EC proliferation may be up-regulated by other growth factors, such as fibroblast growth factor (FGF), hepatocyte growth factor (HGF), and transforming growth factor (TGF)- $\alpha$ and TGF- $\beta$ [20]. VEGF also induces activation and proliferation of HSCs. Then, signaling pathways which determine branching, formation of adequate basement membrane and ECM, and cell migration and differentiation are activated. ECs assemble in tubular structures, whose diameter and length are carefully regulated by VEGF, angiopoietin (Ang)-1, and $\alpha \mathrm{v} \beta 3$ and $\alpha \mathrm{v} \beta 5$ integrins [21]. A structure of vessel network is organized by mechanisms involving signaling pathways that determine branching (ephrins, neuropilin), formation of basement 
membrane and ECM components (MMPs and TIMPs), and cell migration and differentiation [1]. For nascent vessels to mature, pericytes must be recruited [21]. Pericytes are recruited mainly through the release of plateletderived growth factor (PDGF) by ECs [22]. Pericytes release Ang-1, which enables the establishment of adequate junctions between ECs and pericytes, which stabilize nascent vessels [23]. Finally, TGF- $\beta 1$ positively regulates vessel maturation by stimulating the generation of ECM and inducing differentiation of mesenchymal cells into pericytes [24].

\section{Hepatic angiogenesis}

Physiological hepatic angiogenesis during liver regeneration contributes to the formation of new functional sinusoids. Pathological angiogenesis, observed in CLDs, is characterized by the occurrence of capillarized vascular structures and the development of intrahepatic shunts $[1,3]$.

The steps and mechanisms of hepatic angiogenesis mostly overlap with those described for other organs. However, some liver tissue peculiarities are likely to make the process more complicated $[1,11,25]$. The existence of two different types of microvascular structures in the liver-large vessels covered by a continuous endothelium and hepatic sinusoids lined by fenestrated sinusoidal ECs (SECs), which have the ability to produce liver-specific proangiogenic factors, such as angiopoietin-like 3 (ANGPTL3) - may contribute to different pathomechanisms of liver angiogenesis in comparison with the process in other organs. Another liver-specific condition is the presence of HSCs regarded as liver-specific pericytes with a potent profibrogenic activity, which may stimulate angiogenesis, especially in their activated and myofibroblast-like phenotype (HSC/MFs), through mechanisms different from those attributed to microcapillary pericytes $[1,3,11,25$, 26].

From a mechanistic point of view, angiogenesis in CLDs can be interpreted according to two main pathways (Fig. 1). First, neoangiogenesis is stimulated in the hepatic tissue by progressive tissue hypoxia. Most CLDs are characterized by diffuse fibrosis and inflammation. Second, the process of liver chronic wound healing typical for fibrogenic CLDs is characterized by an overexpression of some growth factors, cytokines, and MMPs, with an inherent proangiogenic action [3, 13]. Both phenomena disturb physiological angiogenesis and contribute to structural and functional hepatic disorganization [27]. The accumulation of an excess of ECM with the deposition of fibrillar collagen (type I) instead of physiological sinusoidal collagen (type IV) results in a distorted structure of hepatic sinusoids and loss of specific endothelial fenestration [9]. The deposition of fibrotic tissue evokes resistance to blood flow and impairs the delivery of oxygen to the liver parenchyma.

Inflammatory cells are already present in the liver. Kupffer cells, the resident liver macrophages phagocytize foreign particles to protect hepatic sinusoids. The activation of Kupffer cells leads to the release of cytokines, reactive oxygen species (ROS), and platelet-activating factor (PAF) [13, 28]. PAF induces angiogenesis by the activation of nuclear factor (NF)- $\kappa \mathrm{B}$, which in turn stimulates the production of VEGF [29]. The upregulation of tumor necrosis factor (TNF)- $\alpha$ in a broad variety of cells including macrophages, ECs, mast cells, and adipose tissue fibroblasts results in the activation of the mitogen-activated protein kinase (MAPK)/ERK pathway, a signal transduction pathway involved in cell migration, proteinase induction, regulation of apoptosis, and angiogenesis $[13,30]$. The increase of ROS production in the liver also promotes angiogenesis through overexpression of TNF- $\alpha$, NO, HIF-1, and VEGF [31, 32].

A key area in the study of the cellular and molecular relationship existing between fibrogenesis and angiogenesis concerns the proangiogenic role of activated HSCs/ MFs. HSCs have been suggested to be hypoxia sensitive and cyto- and chemokine-modulated cellular crossroad between inflammatory process, fibrosis, and angiogenesis [11]. Activated HSCs can behave as proangiogenic cells able to react to hypoxia circumstances by the enhancement of VEGF, Ang-1, and their specific receptors VEGFR-2 and Tie-2 (receptor for angiopoietin) [11, 20, 33-35]. On the other hand, HSCs/MFs represent a cellular target for the action of VEGF and Ang-1 [11]. VEGF potentiates HSC proliferation [36], migration, and chemotaxis [20]. The in vivo study showed that $\alpha$-smooth muscle actin ( $\alpha$-SMA)positive HSCs able to express concomitantly VEGF and Ang-1 were found at the leading edge of incomplete and immature fibrotic septa, but not in the established bridging septa. This observation points to the existence of two separate phases of angiogenesis in CLDs [11]. An early phase occurs in the developing septa and is regulated by activated HSCs. A later phase, with expression of proangiogenic agents only in ECs, appears in the large and mature fibrotic septa where the chronic wound healing process is less active and fibrogenic transformation more established [11].

Activated HSCs also stimulate the recruitment of inflammatory cells [33]. It is well known that the inflammatory process stimulates the progression of fibrosis toward the end-point of cirrhosis. Some mediators of the inflammatory response and long-lasting hypoxia activate cells in the surrounding micro-environment to express 
VEGF and other proangiogenic factors to sustain angiogenesis $[37,38]$.

In particular, it should be considered that (1) neovessels themselves may lead to the perpetuation of the inflammatory response expressing chemokines and adhesion molecules promoting the recruitment of inflammatory cells and (2) angiogenesis, early in the course of CLDs, can lead to the transition from acute to chronic inflammation [39].

Angiogenesis in chronic viral hepatitides

Capillary structures formed by ECs in inflamed portal tracts have been observed both in chronic hepatitis $\mathrm{C}$ (CHC) and chronic hepatitis B (CHB) [40, 41]. Evidence of angiogenesis was significantly more frequent in HCVpositive patients compared with $\mathrm{HBV}$-positive patients or controls [40-42]. In addition, HCV-positive sera and liver homogenates stimulated a higher migration and proliferation of human ECs in vitro compared with HBV-positive or control liver sera and liver homogenates [41]. These observations indicate that angiogenesis is particularly linked to $\mathrm{HCV}$ infection, suggesting a possible contribution to HCV-related liver oncogenesis [41, 43]. The intensity of angiogenesis was positively associated with the grade of inflammatory activity in CHC. Evident CD34 expression, which is a marker of neovascularization, was found in the periportal area of lobules and increased in parallel with the fibrosis stage. In the case of septal fibrosis, new-formed blood vessels were observed in the close neighborhood of fibrous septa [5, 6]. The molecular mechanisms involved in chronic viral hepatitis-associated angiogenesis have not been fully identified. Hepatic VEGF expression was found to be significantly higher in stages 3 and 4 compared with stages 1 and 2 of fibrosis and revealed a positive correlation with the fibrosis stage [4]. Similarly, the hepatic expression of HGF is up-regulated in the course of chronic viral hepatitis [44, 45]. HGF induces EC proliferation in a VEGF-dependent manner [45]. Additionally, expression of PDGF in macrophages and infiltrating inflammatory cells and PDGF receptors in sinusoidal and perisinusoidal cells in periportal areas is increased during chronic viral hepatitis. These alterations facilitate the stabilization of nascent blood vessels [2, 46]. Overexpression of inducible NO synthase in the livers of patients with $\mathrm{CHC}$ or $\mathrm{CHB}$ results in the overproduction of $\mathrm{NO}$ which participate in the angiogenic response by inducing vasodilatation [47].

Another phenomenon that is involved in fibrosis progression and increases hepatocellular vulnerability to necrosis in the course of CHC is steatosis [48]. The fibrogenic effect of steatosis is mediated by increased oxidative stress and insulin resistance, induction of apoptosis, and activation of HSCs and proinflammatory cytokines [48]. Hepatocyte injury, due to steatosis, evokes reparative and regeneration processes. The wound healing process is strictly associated with new blood vessel formation. All analyzed CHC patients with steatosis had a significantly higher CD34 expression both in portal tracts and fibrous septa and lobules [6]. The intensity of angiogenesis was positively associated with the grade of steatosis. In the case of more advanced steatosis, evident CD34 expression was observed in steatotic areas of lobules and was localized mainly in the periportal zone [6].

CD31+ and cadherin 5+ ECs with microvessel morphology were observed in inflamed portal tracts and more evident in more inflamed portal tracts of CHC patients, acquiring a characteristic form of capillary tube formation [40]. This observation additionally supports an interaction between the inflammatory activity and neoangiogenesis. It is possible that activated cytotoxic $\mathrm{T}$ cells may up-regulate the expression of vascular adhesion molecules, which enhance angiogenesis [21]. The new vessels are an integral part of tissue remodeling that accompanies chronic inflammation and provide a portal of entry for the continuing recruitment of inflammatory cells. They also deliver nutrients and oxygen to areas of chronic inflammation that are relatively hypoxic [49].

One highly specialized form of tissue remodeling in chronic inflammation is lymphoid neogenesis, the development of new lymphoid tissue in inflammatory sites at times when normal lymph node development is complete. These inflammatory lymphoid follicles provide a microenvironment for the recruitment and retention of lymphocytes at sites of chronic inflammation. In $\mathrm{CHC}$, new CD34+ vessels are found in areas of lymphoid neogenesis which occurs in response to local cytokines in the portal tract to form expanded portal-associated lymphoid tissue $[5,49,50]$.

$\mathrm{CHC}$ patients present a proangiogenic profile of angiogenesis soluble markers [51, 52]. Serum VEGF, Ang-2, and Tie-2 are increased in viral hepatitis and their concentrations could be valuable markers of the evolution of liver inflammation, disease progression, and response to therapy [51]. Treatment of $\mathrm{CHC}$ patients with pegylated interferon and ribavirin significantly reduces inflammatory process and therefore decreases VEGF and Ang-2 concentration [51, 52].

Angiogenesis in nonalcoholic fatty liver disease

NAFLD encompasses a wide spectrum of liver tissue alterations ranging from pure steatosis, through NASH to cirrhosis and hepatocellular carcinoma (HCC) [53]. The severe form of NAFLD is NASH given its progressive disease course and considering that NASH can evolve from steatohepatitis to fibrosis, cirrhosis, and can eventually lead to HCC [52]. The mechanism of NASH is still not fully 
explained. The pathophysiology of NASH should be recognized as a multifactorial process [54]. In some stages of NASH, a link can be made between the disease progression and hepatic microvasculature changes. Fat accumulation damages hepatocytes leading to the deregulation of the microvascular flow and lipotoxicity. Reduction in sinusoidal perfusion initially arises from the effects of hepatocytes swollen with accumulated lipids. This results in the reduction of the intrasinusoidal volume, altering the sinusoidal architecture [11]. Lipotoxicity activates cytokines, which may induce the recruitment of inflammatory cells. Moreover, inflammation triggers vascular permeability and increases the migration of monocytes, macrophages, mast cells, platelets, and lymphocytes. These cells are able to initiate angiogenesis through various pathways. NASH is characterized by adhesion of leukocytes to SECs, followed by leukocyte infiltration into the hepatic parenchyma to form inflammatory foci $[52,55]$. Enlarged, fat-laden, and inflamed hepatocytes, together with perivascular fibrosis, narrow the sinusoidal lumens, making vessels more tortuous and impairing sinusoidal perfusion [55]. This chronic liver damage might initiate hepatic angiogenesis.

Some studies showed a significant development of hepatic CD34-positive neovascularization in NASH, whereas almost no development was observed in simple steatosis. The degree of angiogenesis was almost parallel to that of liver fibrosis in NASH [56]. These results were consistent with a previous finding that angiogenesis increases stepwise during hepatic fibrosis development in several models of fibrosis, including the rodent dietary NASH model [57]. An important issue was to identify the localization of neovascularization during the progression of NASH. CD34-positive neovessels were initially observed around the central vein (zone III) and then gradually progressed to the portal area (zone I). They were also observed along the fibrotic septa in high-grade fibrosis $[56,57]$. These progression patterns are similar to those of fibrosis development in NASH and it is likely that neovascularization in NASH progresses almost concurrently with fibrosis development and plays a pivotal role in the onset and progression of NASH [57]. Another intriguing finding was that angiogenesis is triggered differently in both morbidly obese and nonobese NAFLD patients [7]. In morbidly obese individuals, angiogenesis was independent of NASH and was activated at an early stage-(simple steatosis), whereas in nonobese patients, it was activated at the level of NASH [7]. The development of NASH in severely obese patients had no influence on the hepatic expression of angiogenic factors. However, these factors were positively correlated with the severity of fibrosis. This observation clearly indicates that the development of fibrosis is preceded by angiogenesis that is independent of NASH [7]. A macroarray gene expression analysis of the liver of obese patients with severe
NASH revealed that VEGF, TGF- $\beta 1$, connective tissue growth factor, and fibroblast growth factor were overexpressed compared with controls [58].

Association between angiogenesis and adipokines in CLDs

Among viral hepatitides, HCV infection has been documented to show more metabolic abnormalities. NAFLD is a common manifestation of metabolic syndrome. Since CHC and NAFLD have been recognized not only as inflammatory diseases but metabolic diseases as well, various adipocytokines (adipokines) - adipose tissuederived cytokines-are considered to be involved in their progression [47, 57, 59-61]. Chronic inflammation, obesity, and insulin resistance result in an altered adipokine profile. Adipokines have been shown not only to be regulators of fibrogenesis and metabolic and inflammatory processes but also as potent regulators of angiogenesis. They may influence structures and regulate the synthesis of agents responsible for the modulation of angiogenesis [47, 57, 60]. Leptin, visfatin/PBEF-1/Nampt (pre-B cell colony-enhancing factor $1 /$ nicotinamide phosphoribosyltransferase), chemerin (also known as retinoic acid receptor responder protein 2 (RARRES2), tazarotene-induced gene two protein (TIG2) or RAR-responsive protein (TIG2), and resistin have been found to promote angiogenesis, whereas adiponectin attenuates it $[33,57,61-65]$. Up to now, there has only been one study that has described the influence of vaspin (visceral adipose tissue-derived serine protease inhibitor, SERPINA12) on ECs [66].

Leptin enhances the proliferation of activated HSCs, which play a central role in liver fibrosis development $[47,59,60]$. In addition to these direct effects on HSCs, studies have revealed that leptin possesses a proangiogenic activity coordinated with VEGF $[47,57]$ and leptin-mediated neovascularization plays an important role in the development of liver fibrosis in the rat NASH model [57]. The activation of leptin receptors in HSCs leads to an increase in VEGF expression [19]. Moreover, leptin is able to trigger a ERK1/2- and PI3K-dependent nuclear translocation of HIF-1 $\alpha$ [33].

Serum leptin level was significantly higher in NASH compared wtih patients with simple steatosis [57]. In CHC, serum leptin was significantly increased in CHC compared with healthy controls and correlated positively with the grade of inflammatory activity [44]. Other authors reported its positive association with the fibrosis stage and higher levels in the case of cirrhosis [67]. These results suggest that, as in the animal model, leptin-mediated angiogenesis plays some role in the progression of NASH and CHC.

New adipokines such as visfatin and chemerin have been found to exert proangiogenic activities [62, 63]. 
Increased levels of those adipokines were observed in NAFLD and CHC [68-71]. There is only one study that has assessed the relationship between angiogenesis and novel adipokines in $\mathrm{CHC}$ [72]. The study showed surprisingly that visfatin was negatively associated with the angiogenesis intensity only in females, but not in males. Another novel adipokine vaspin was significantly down-regulated in $\mathrm{CHC}$ patients, but raised in the case of more advanced fibrosis [71]. It was positively associated with neovessel formation in those patients. The strongest relationship was observed in males [72]. These results suggest that the role of some adipokines in angiogenesis may differ in both males and females.

\section{Conclusions}

In conclusion, angiogenesis and related changes in the angioarchitecture have been proposed to potentiate fibrosis progression toward cirrhosis. Angiogenesis in liver is characterized by intrahepatic vascular remodeling with capillarization of the sinusoids and development of intrahepatic shunts, which leads to increased hepatic resistance and decreased effective hepatocyte perfusion. Hypoxia and HIF-related cellular responses are emerging as crucial in CLD progression. Some cellular and molecular mechanisms have been described which regulate the cross-talk between angiogenesis and fibrogenesis and various hepatic cell populations. Hypoxic hepatocytes, SECs, and activated HSCs play a major role. HSCs are believed to represent a crucial crossroad at the interaction between inflammation, angiogenesis, and fibrogenesis. VEGF is the most potent proangiogenic factor and is up-regulated in CLDs. Metabolic abnormalities, i.e., steatosis and adipose tissuederived cytokines, seem to influence inflammatory activity, fibrogenesis, and angiogenesis.

The study by Ciupińska-Kajor et al. [7] revealed that in morbidly obese patients, angiogenesis was independent of NASH and was activated early in the natural history of NAFLD. Moreover, angiogenesis was strictly associated with stage of hepatic fibrosis in those patients. These new data suggest that angiogenesis may precede hepatic fibrosis development and then aggravate its progression. These observations imply possible way of tumorigenesis in NAFLD without cirrhosis.

Clinical trials of appropriate design and on a large number of patients with primary end-points are needed. The proposed association between angiogenesis, chronic wound healing, and fibrosis progression indicate for the following potential clinical goals: (1) measurement of proangiogenic factors may serve as a noninvasive way to monitor both disease progression as well as the response to therapy; (2) antiangiogenic therapy may be an effective tool for inhibiting or attenuating the progression of fibrosis in CLDs. However, antiangiogenic agents ought to be used with caution and be carefully balanced in patients with CLDs due to the fact that angiogenesis is a relevant phenomenon in wound healing and excessive blocking of angiogenesis may not represent the desired therapeutic objective. Moreover, the angiogenic effect might be just one manifestation and consequence of the underlying multiple causes of CLDs.

\section{References}

1. Medina J, Arroyo AG, Sanchez-Madrid F, Moreno-Otero R. Angiogenesis in chronic inflammatory liver disease. Hepatology 2004;39:1185-1195.

2. Chaparro M, Sanz-Cameno P, Trapero-Marugan M, Garcia-Buey L, Moreno-Otero R. Mechanisms of angiogenesis in chronic inflammatory liver disease. Ann Hepatol 2007;6:208-213.

3. Kukla M, Gabriel A, Waluga M, Bułdak R, Mazur W. Angiogenesis in chronic viral hepatitis. Gastroenterol Pol 2009;16(4):304-309.

4. Amarapurkar AD, Amarapurkar DN, Vibhav S, Patel ND. Angiogenesis in chronic liver disease. Ann Hepatol 2007;6:170-173.

5. Gabriel A, Kukla M, Wilk M, et al. Angiogenesis in chronic hepatitis $\mathrm{C}$ is associated with inflammatory activity grade and fibrosis stage. Pathol Res Pract 2009;205:758-764.

6. Kukla M, Gabriel A, Sabat D, et al. Association between liver steatosis and angiogenesis in chronic hepatitis C. Pol J Pathol 2010;3:154-160.

7. Ciupińska-Kajor M, Hartleb M, Kajor M, et al. Hepatic angiogenesis and fibrosis are common features in morbidly obese patients. Hepatol Int 2011; doi:10.1007/s12072-011-9320-9.

8. Pugh CW, Rateliffe PJ. Regulation of angiogenesis by hypoxia: role of the HIF system. Nat Med 2003;9:677-684.

9. Sanz-Cameno P, Trapero-Marugan M, Chaparro M, Jones EA, Moreno-Otero R. Angiogenesis: from chronic liver inflammation to hepatocellular carcinoma. J Oncol 2010; doi:10.1155/2010/ 272170.

10. Simpson KJ, Henderson NC, Bone-Larson CL, et al. Chemokines in the pathogenesis of liver disease: so many players with poorly defined roles. Clin Sci 2003;104(1):47-63.

11. Paternostro C, David E, Novo E, Parola M. Hypoxia, angiogenesis and liver fibrogenesis in the progression of chronic liver diseases. World J Gastroenterol 2010;16(3):281-288.

12. Noonan DM, De Lerma Barbaro A, Vannini N, Mortara L, Albini A. Inflammation, inflammatory cells and angiogenesis: decisions and indecisions. Cancer Metastasis Rev 2008;27(1):31-40.

13. Coulon S, Heindryckx F, Geerts A, et al. Angiogenesis in chronic liver disease and its complication. Liver Int 2011;31(2):146-162.

14. Murohara T, Asahara T, Silver M, et al. Nitric oxide synthase modulates angiogenesis in response to tissue ischemia. J Clin Invest 1998;101:2567-2578.

15. Jackson C. Matrix metalloproteinases and angiogenesis. Curr Opin Nephrol Hypertens 2002;11:295-299.

16. Hynes RO. A reevaluation of integrins as mediators of angiogenesis. Nat Med 2002;8:918-921.

17. Galvez BG, Matias-Roman S, Albar J, Sanchez-Madrid F, Arroyo A. Membrane type 1-matrix metalloproteinase is activated during migration of human endothelial cells and modulates endothelial 
motility and matrix remodeling. J Biol Chem 2001;276:3729137500 .

18. Luttun A, Dewerchin M, Collen D, Carmeliet P. The role of proteinases in angiogenesis, heart development, restenosis, atherosclerosis, myocardial ischemia, and stroke: insights from genetic studies. Curr Atheroscler Rep 2000;2:497-516.

19. Ferrara N, Gerber H, LeCouter J. The biology of VEGF and its receptors. Nat Med 2003;9:669-676.

20. Novo E, Cannito S, Zamara E, et al. Proangiogenic cytokines as hypoxia dependent factors stimulating migration of human hepatic stellate cells. Am J Pathol 2007;170(6):1942-1953.

21. Jain RK. Molecular regulation of vessel maturation. Nat Med 2003;9:685-693.

22. Hellstrom M, Gerhardr H, Kalen M, et al. Lack of pericytes leads to endothelial hyperplasia and abnormal vascular morphogenesis. J Cell Biol 2001;153:543-553.

23. Thurston G, Rudge J, Ioffe E, et al. Angiopoietin-1 protects the adult vasculature against plasma leakage. Nat Med 2000;6:460-463.

24. Goumans MJ, Valdimarsdottir G, Itoh S, et al. Balancing the activation state of the endothelium via two distinct TGF-beta type I receptors. EMBO J 2002;21(7):1743-1753.

25. Fernández M, Semela D, Bruix $J$, et al. Angiogenesis in liver disease. J Hepatol 2009;50:604-620.

26. Camenisch G, Pisabarro M, Sherman D, et al. ANGPTL3 stimulates endothelial cell adhesion and migration via integrin alpha $\mathrm{v}$ beta 3 and induces blood vessel formation in vivo. J Biol Chem 2002;227:17281-1790.

27. Thorgeirsson SS, Grisham JW. Molecular pathogenesis of human hepatocellular carcinoma. Nat Genet 2003;17(1):1-16.

28. Horie Y, Wolf R, Russel J, Shanley TP, Granger DN. Role of Kupffer cells in ischaemia/reperfusion-induced hepatic microvascular dysfunction in mice. Hepatology 1997;26:1499-1505.

29. Ko HM, Seo KH, Han SJ, et al. Nuclear factor kappaB dependency of platelet-activating factor-induced angiogenesis. Cancer Res 2002;62:1809-1814.

30. Huang Y, Li S. Detection of characteristic sub pathway network for angiogenesis based on the comprehensive pathway network. BMC Bioinformatics 2010;11(Suppl.1):S32.

31. Dewhirst MW, Cao Y, Moeller B. Cycling hypoxia and free radicals regulate angiogenesis and radiotherapy response. Nat Rev Cancer 2008;8:425-437.

32. Novo E, Parola M. Redox mechanisms in hepatic chronic wound healing and fibrogenesis. Fibrogenesis Tissue Repair 2008;13:5.

33. Aleffi S, Petrai I, Bertolani C, et al. Upregulation of proinflammatory and proangiogenic cytokines by leptin in human hepatic stellate cells. Hepatology 2005;42:1339-1348.

34. Wang YQ, Luk JM, Ikeda K, et al. Regulatory role of vHL/HIF-1 alpha in hypoxia induced VEGF production in hepatic stellate cells. Biochem Biophys Res Commun 2004;317:358-362.

35. Ankoma-Sey V, Wang Y, Dai Z. Hypoxic stimulation of vascular endothelial growth factor expression in activated rat hepatic stellate cells. Hepatology 2000;31:141-148.

36. Olaso E, Salado C, Egilegor E, et al. Proangiogenic role of tumoractivated hepatic stellate cells in experimental melanoma metastasis. Hepatology 2003;37:674-685.

37. Copple BL, Bustamante JJ, Welch TP, Kim ND, Moon JO. Hypoxia-inducible factor-dependent production of profibrotic mediators by hypoxic hepatocytes. Liver Int 2009;29:1010-1021.

38. Carmeliet P. Angiogenesis in health and disease. Nat Med 2003;9:653-660.

39. Jackson JR, Seed MP, Kircher CH, Willoughby DA, Winkler JD. The codependence of angiogenesis and chronic inflammation. FASEB J 1997;11:457-465.

40. Garcia-Monzon C, Sanchez-Madrid F, Garcia-Buey L, et al. Vascular adhesion molecule expression in viral chronic hepatitis: evidence of neoangiogenesis in portal tracts. Gastoenterology 1995;108:231-241.

41. Mazzanti R, Messerini L, Monsacchi L, et al. CVH induced by hepatitis C but not hepatitis B virus infection correlates with increased liver angiogenesis. Hepatology 1997;25:229234.

42. Messerini L, Novelli L, Comin CE. Microvessel density and clinicopathological characteristics in hepatitis $\mathrm{C}$ virus and hepatitis B virus related hepatocellular carcinoma. J Clin Pathol 2004;57:867-871.

43. Ohmori S, Shiraki K, Sugimoto K, et al. High expression of CD34-positive sinusoidal endothelial cells is risk factor for hepatocellular carcinoma in patients with $\mathrm{HCV}$-associated chronic liver diseases. Hum Pathol 2001;32:1363-1370.

44. Żwirska-Korczala K, Kukla M, Ziółkowski A, et al. Leptin, neopterin and hepatocyte growth factor as markers of fibrosis and inflammatory activity in chronic hepatitis C. Exp Clin Hep 2005;1:OR60-OR65.

45. Medina J, Caveda L, Sanz-Cameno P, et al. Hepatocyte growth factor activates endothelial proangiogenic mechanisms relevant in chronic hepatitis C-associated neoangiogenesis. J Hepatol 2003;35:660-667.

46. Ikura Y, Morimoto H, Ogami M, et al. Expression of platelet derived growth factor and its receptor in livers of patients with chronic liver disease. J Gastroenterol 1997;32:496-501.

47. Kukla M, Mazur W, Bułdak RJ, Żwirska-Korczala K. Potential role of leptin, adiponectin and three novel adipokines-visfatin, chemerin and vaspin-in chronic hepatitis. Mol Med 2011; 17(11-12):1397-1410.

48. Majano PL, Garcia-Monzon C, Lopez-Cabrera M, et al. Inducible nitric oxide synthase expression in chronic viral hepatitis. Evidence for a virus-induced gene upregulation. J Clin Invest 1998;101:1343-1352.

49. Lai WK, Adams DH. Angiogenesis and chronic inflammation; the potential for novel therapeutic approaches in chronic liver disease. J Hepatol 2005;45:7-11.

50. Girard JP, Springer TA. High endothelial venules (HEVs): specialized endothelium for lymphocyte migration. Immunol Today 1995;16:449-457.

51. Salcedo X, Medina J, Sanz-Cameno P, et al. The potential of angiogenesis soluble markers in chronic hepatitis C. Hepatology 2005;42:696-701.

52. Salcedo X, Medina J, Sanz-Cameno P. Review article: angiogenesis soluble factors as liver disease markers. Aliment Pharmacol Ther 2005;22:23-30.

53. Bellentani S, Marino M. Epidemiology and natural history of non-alcoholic fatty liver disease (NAFLD). Ann Hepatol 2009;8(Suppl. 1):S4-S8.

54. Sanal MG. The blind men 'see' the elephant: the many faces of fatty liver disease. World J Gastroenterol 2008;14(6):831-844.

55. McCuskey RS, Ito Y, Robertson GR, et al. Hepatic microvascular dysfunction during evolution of dietary steatohepatitis in mice. Hepatology 2004;40:386-393.

56. Kitade M, Yoshiji H, Noguchi R, et al. Crosstalk between angiogenesis, cytokeratin-18, and insulin resistance in the progression of non-alcoholic steatohepatitis. World J Gastroenterol 2009;15:5193-5199.

57. Kitade M, Yoshiji H, Kojima H, et al. Leptin-mediated neovascularization is a prerequisite for progression of nonalcoholic steatohepatitis in rats. Hepatology 2006;44:983-991.

58. Cayon A, Crespo J, Guerra AR, Pons-Romero F. Gene expression in obese patients with non-alcoholic steatohepatitis. Rev Esp Enferm Dig 2008;100:212-218.

59. Marra F, Bertolani C. Adipokines in liver diseases. Hepatology 2009;50:957-969. 
60. Bertolani C, Marra F. The role of adipokines in liver fibrosis. Pathophysiology 2008;15:91-101.

61. Anagnostoulis S, Karayiannakis AJ, Lambropoulou M, et al. Human leptin induces angiogenesis in vivo. Cytokine 2008;42: 353-357.

62. Adya R, Tan BK, Punn A, et al. Visfatin induces human endothelial VEGF and MMP-2/9 production via MAPK and PI3 K/ Akt signalling pathways: novel insights into visfatin- induced angiogenesis. Cardiovasc Res 2008;78:356-365.

63. Kaur J, Adya R, Tan BK, et al. Identification of chemerin receptor (ChemR23) in human endothelial cells: chemerininduced endothelial angiogenesis. Biochem Biophys Res Commun 2010;391:1762-1768.

64. Robertson SA, Rae CJ, Graham A. Induction of angiogenesis by murine resistin: putative role of PI3-kinase and NO-dependent pathways. Regul Pept 2009;152:41-47.

65. Brakenhielm E, Veitonmäki N, Cao R, et al. Adiponectin-induced antiangiogenesis and antitumor activity involve caspase mediated endothelial cell apoptosis. Proc Natl Acad Sci USA 2004;101: 2476-2481.

66. Fu BD, Yamawaki H, Okada M, Hara Y. Vaspin cannot inhibit TNF-alpha-induced inflammation of human umbilical vein endothelial cells. J Vet Med Sci 2009;71:1201-1207.
67. Piche T, Vandenbos F, Abakar-Mahamat G, et al. The severity of liver fibrosis is associated with high leptin levels in chronic hepatitis C. J Viral Hepatol 2004;11:91-96.

68. Jarrar MH, Baranova A, Collantes R, et al. Adipokines and cytokines in non-alcoholic fatty liver disease. Aliment Pharmacol Ther 2008;27(5):412-421.

69. Kukla M, Żwirska-Korczala K, Hartleb M, et al. Serum chemerin and vaspin in nonalcoholic fatty liver disease. Scan J Gastroenterol 2010;45(2):235-242.

70. Kukla M, Żwirska-Korczala K, Gabriel A, et al. Visfatin serum levels in chronic hepatitis C patients. J Viral Hepat 2010;17(4): 254-260.

71. Kukla M, Żwirska-Korczala K, Gabriel A, et al. Chemerin, vaspin and insulin resistance in chronic hepatitis C. J Viral Hepat 2010;17(9):661-667.

72. Kukla M, Berdowska A, Gabriel A, et al. Association between hepatic angiogenesis and serum adipokine profile in non-obese chronic hepatitis C patients. Pol J Pathol 2011;4:218-228. 